\title{
Star-forming regions as potential contributors to Galactic cosmic rays: the case of NGC 3603
}

\section{Lab Saha, ${ }^{a, *}$ Alberto Dominguez, ${ }^{a}$ Luigi Tibaldo, ${ }^{b}$ Marco Ajello ${ }^{c}$ and Marianne Lemoine-Goumard $^{d}$ on behalf of the Fermi-LAT Collaboration}

a IPARCOS and Department of EMFTEL, Universidad Complutense de Madrid, E-28040 Madrid, Spain

${ }^{b}$ IRAP, Universite de Toulouse, CNRS, UPS, CNES, Toulouse, France

${ }^{c}$ Department of Physics and Astronomy, Clemson University, Kinard Lab of Physics, Clemson, SC 29634-0978, USA

${ }^{d}$ Centre d' Etudes Nucléaires de Bordeaux Gradignan, IN2P3/CNRS, Université de Bordeaux, BP120, F-33175 Gradignan Cedex, France

E-mail: labsaha@ucm.es, alberto.d@ucm.es, luigi.tibaldo@irap.omp.eu, majello@g.clemson.edu, lemoine@cenbg.in2p3.fr

The identification of the sources contributing to the acceleration of Galactic Cosmic Rays (CRs) is a long-standing puzzle. Star-forming regions (SFRs) may be one of these potential contributors, in fact, the detection of $\gamma$-rays from the Cygnus Cocoon indicates the existence of freshly accelerated high-energy particles in the region, making it the first case of a firm detection of CR acceleration in SFRs. However, the limited number of such $\gamma$-ray detections are preventing any conclusion about the prevalence of SFRs as CR sources. In this paper, we present a summary of the morphological and spectral study of the unidentified source 4FGL J1115.1-6118 using about ten years of data above $10 \mathrm{GeV}$ taken with Fermi-LAT. This source is positionally coincident with the young massive stellar cluster NGC 3603, and represents one of the few cases already studied in $\gamma$-rays. We will also present perspectives for a systematic search of $\gamma$-ray emitting SFRs. These are the first steps towards potentially establishing these types of sources as fundamental CR emitters. The results included in this proceeding and shown in this presentation were published in ref. [1]. The details about the analysis, interpretation and modelling of 4FGL J1115.1-6118 can be found in the aforementioned reference.

$37^{\text {th }}$ International Cosmic Ray Conference (ICRC 2021)

July 12 th - 23rd, 2021

Online - Berlin, Germany

\footnotetext{
${ }^{*}$ Presenter
} 
The identification of major contributors to the observed Cosmic Rays (CRs) fluxes is a prime objective towards the resolution of the long-standing enigma of the origin of CRs. Supernova remnants (SNRs) are regarded as promising sources of Galactic CRs with energies up to the $10^{15}$ $\mathrm{eV}$ (the knee of the CR spectrum). However, over the last decade, space- and ground-based telescopes have revealed new classes of Galactic $\gamma$-ray source populations. Some of them can be considered as complementary or alternative CR sources.

Star-forming regions (SFRs) are considered potential contributors to the acceleration of Galactic cosmic rays, and detection of $\gamma$-rays from such SFRs in our Galaxy can establish the presence of relativistic charged particles. Indeed, the detection of $\gamma$-rays from the Cygnus cocoon indicates the presence of freshly accelerated high-energy particles in the SFR, making it the first case of a firm detection of such cosmic-ray acceleration [3].

$\gamma$-ray emission is also detected from a few more massive stellar cluster such as Westerlund 1 $[4,5]$ and NGC 3603 [1, 6]. In a search for such sources, recently some more studies have been performed [7-9]. All these evidences make SFRs an interesting class of $\gamma$-ray candidates for CR accelerators. Therefore, SFRs have become a new class of candidates for CR accelerators [2]. However, several questions are still open, and more observational evidence is required to establish the role of SFRs in the CR lifecycle.

NGC 3603 is an SFR that has been observed at various wavebands, including $\gamma$-rays. NGC 3603 is a nebula situated in the Carina spiral arm of the Milky Way at a distance of about $7 \pm 1$ $\mathrm{kpc}$ from the solar system [10-15]. It is a massive $\left(\mathrm{M}>2000 M_{\odot}\right)$ HII region and one of the most luminous optically visible ones in the Milky Way, being powered by a cluster of OB stars [16]. The H-alpha luminosity of NGC 3603 is $L\left(H_{\alpha}\right) \sim 1.5 \times 10^{39} \mathrm{ergs} \mathrm{s}^{-1}$ [17], and the total mass of molecular clouds is about $4.1 \times 10^{5} \mathrm{M}$ [18]. The average age of the cluster is between 2 and $3 \mathrm{Myr}$ and the star formation in and around the young cluster has been going on for about 10-20 Myr $[10,15]$.

Recently, we have investigated 4FGL J1115.1-6118 using about 10 years of Fermi-LAT data. This source positionally coincident with NGC 3603 was first detected in the third Fermi-LAT Hard Source Catalog [3FHL J1115-6117; 19]. Earlier studies above $10 \mathrm{GeV}$ from the same region using 7 years of Fermi-LAT data suggested the presence of extended emission [6]. The emission was claimed to have a hard spectrum with a photon index of $2.3 \pm 0.1$ from $1 \mathrm{GeV}$ to $250 \mathrm{GeV}$. The emission region was best-fitted with a Gaussian centred at RA $(\mathrm{J} 2000)=167^{\circ} .8 \pm 0^{\circ} .1$, Dec $(\mathrm{J} 2000)=-61^{\circ} .3 \pm 0^{\circ} .1$ with width $=1^{\circ} .1 \pm 0^{\circ} .1$, corresponding to a significance of more than $10 \sigma$. Taking advantage of an extended Fermi-LAT dataset with better event-level analysis and improved interstellar emission models [20], along with X-ray data, we studied emission from the fourth Fermi-LAT catalog source 4FGL J1115.1-6118.

The results of our detailed analysis show that the observed $\gamma$-ray emission from the 4FGL J1115.1-6118 source is not significantly extended as reported before. We remarked that this region also contains the extended source FGES J1109.4-6115e, which plays a significant role in characterizing the morphological properties of the sources present in this region and deserves further analysis. Observed X-ray emission was also observed to be associated with the point source 4FGL J1115.1-6118 and Galactic in origin. We also found that the observed SED could be explained with both a leptonic and a hadronic model for density of ambient matter of $35 \mathrm{~cm}^{-3}$.

We investigated all possible association of the detected source with the known classes of $\gamma$-ray 
emitters. However, no such association can be made. Therefore we speculate that 4FGL J1115.16118 is a case of $\gamma$-ray emitting SFR. Hence, it becomes a potential candidate for studying SFRs to understand the origin of cosmic rays using the next generation of $\gamma$-ray telescopes such as the Cherenkov Telescope Array.

Interstellar background modelling is a paramount source of uncertainty in the characterization of emission from Galactic sources, especially extended ones. Hence we plan to select a few alternate interstellar background models to assess the related uncertainties, Finally, to establish this class of sources as a potential contributor to galactic CR, it is highly desirable to extend $\gamma$-ray studies to a larger sample of SFRs which might be associated with emission of $\gamma$-rays. Thus we also plan to perform a systematic search of $\gamma$-ray emission from a large sample of stellar clusters. The detection of more $\gamma$-ray sources from the direction of SFRs or the non-detection of many targets will tell us about this class of sources as possible sites of Galactic CR acceleration depending on their properties such as age and stellar content.

\section{Acknowledgement:}

Fermi-LAT collaboration

\section{References}

[1] Saha, L., Domínguez, A., Tibaldo, L., et al. 2020, ApJ, 897, 131

[2] Aharonian, F., Yang, R., \& de Oña Wilhelmi, E. 2019, Nature Astronomy, 3, 561

[3] Ackermann, M., Ajello, M., Allafort, A., et al. 2011, Science, 334, 1103

[4] Abramowski, A., Acero, F., Aharonian, F., et al. 2012, A\&A, 537, A114

[5] Ohm, S., Hinton, J. A., \& White, R. 2013, MNRAS, 434, 2289

[6] Yang, R.-z., \& Aharonian, F. 2017, A\&A, 600, A107

[7] Yang, R.-z., de Oña Wilhelmi, E., \& Aharonian, F. 2018, AA, 611, A77

[8] Liu, B., Yang, R.-. zhi ., Sun, X.-. na ., et al. 2019, ApJ, 881, 94

[9] Katsuta, J., Uchiyama, Y., \& Funk, S. 2017, ApJ, 839, 129

[10] Beccari, G., Spezzi, L., De Marchi, G., et al. 2010, ApJ, 720, 1108

[11] Crowther, P. A., \& Dessart, L. 1998, MNRAS, 296, 622

[12] de Pree, C. G., Nysewander, M. C., \& Goss, W. M. 1999, AJ, 117, 2902

[13] Nürnberger, D. E. A., \& Petr-Gotzens, M. G. 2002, A\&A, 382, 537

[14] Pandey, A. K., Ogura, K., \& Sekiguchi, K. 2000, PASJ, 52, 847

[15] Melnick, J., Tapia, M., \& Terlevich, R. 1989, A\&A, 213, 89

[16] Goss, W. M., \& Radhakrishnan, V. 1969, ApJ Letter, 4, 199 
[17] Kennicutt, R. C., J. 1984, APJ, 287, 116

[18] Grabelsky, D. A., Cohen, R. S., Bronfman, L., \& Thaddeus, P. 1988, ApJ, 331, 181

[19] Ajello, M., Atwood, W. B., Baldini, L., et al. 2017, , 232, 18

[20] Abdollahi, S., Acero, F., Ackermann, M., et al. 2020, The Astrophysical Journal Supplement Series, 247, 33. https: //doi .org/10.3847\%2F 1538-4365\%2Fab6bcb 